\title{
Clinical Study on the Intervention of Coptis Root and Ginseng on gut microbiota in Type 2 Diabetes: a study protocol for a randomized controlled and double-blinding trial
}

\section{Li Jiang ( $\sim 294143396 @ q q . c o m$ )}

Beijing University of Chinese Medicine https://orcid.org/0000-0003-3827-2178

\section{Qiang Fu}

Beijing University of Chinese Medicine

\section{Weijun Huang}

Beijing University of Chinese Medicine

Yu Chen

Beijing University of Chinese Medicine Jiayue Li

Beijing University of Chinese Medicine Yonghua Xiao

Beijing University of Chinese Medicine

Ruixi Sun

Beijing University of Chinese Medicine

\section{Esther Aijia Shen}

Beijing University of Chinese Medicine

\section{Junheng Wang}

Beijing University of Chinese Medicine

Yaofu Zhang

Beijing University of Chinese Medicine

Zhuang Li

Beijing University of Chinese Medicine Jiangteng Liu

Beijing University of Chinese Medicine

Xiaozhe Fu

Beijing University of Chinese Medicine

Yuanyuan Liu

Beijing University of Chinese Medicine

\section{Yu Zhao}

Beijing University of Chinese Medicine 


\section{Guanxun Su}

Beijing University of Chinese Medicine

\section{Shidong Wang}

Beijing University of Chinese Medicine Jinxi Zhao

Beijing University of Chinese Medicine

\section{Research Article}

Keywords: protocol, Type 2 Diabetes, TCM, gut microbiota, Randomized controlled trial

Posted Date: September 2nd, 2021

DOl: https://doi.org/10.21203/rs.3.rs-839673/v2

License: (c) (1) This work is licensed under a Creative Commons Attribution 4.0 International License.

Read Full License 


\section{Abstract}

Background: Type 2 Diabetes Mellitus (T2DM) is a complex metabolic disease caused by complicated factors, the pathogenesis of which has not been determined. Traditional Chinese medicine(TCM) could effectively regulate blood sugar and influence gut microbiota in T2DM patients. Preliminary studies showed that the coptis root and ginseng (RG) formula could relieve insulin resistance and regulate the progression of diabetes in mice. The purpose of this study is to explore the efficacy and safety of RG formula in the treatment of adult patients with T2DM, as well as observing its effects on gut microbiota.

Methods and analysis: This trial is a randomized, double-blind, placebo-controlled study. A total of 60 participants will be randomized in a 1:1 ratio into an experiment group (RG formula) and a control group (placebo). Patients in both groups will be given diabetes education and basic blood glucose control. Glucose-lowering drugs with significant influence on gut microbiota will be avoided. This trial will last 25 weeks including 1 week run-in, 12 weeks intervention and 12 weeks follow-up visit. The primary outcome is the change in the $\mathrm{HbA} 1 \mathrm{c}$. The secondary outcomes comprise the change in the fasting blood glucose(FBG), postprandial blood glucose(PBG) fasting insulin(FIL), fasting C-peptide(C-P), insulin resistance index(IRI), inflammatory factors and species abundance of gut microbiota between the two groups. Safety in medication will also be evaluated. The correlation analysis will be explored between the glycemic indicators,

inflammatory factors and abundance of gut microbiota.

Discussion: This study will provide the clinical evidence for the efficacy of RG formula in regulating blood sugar and influencing gut microbiota, which will be beneficial to form the integrated therapeutic regimen in T2DM with TCM.

Trial registration: "Clinical Study on the Intervention of Coptis Root and Ginseng", Chinese Clinical Trials Registry ChiCTR 2100042126. Registered on 14 January, 2021,

http://www.chictr.org.cn/edit.aspx?pid=42669\&htm=4

\section{Background}

Diabetes Mellitus(DM) is a group of endocrine and metabolic diseases characterized by hyperglycemia, which is caused by absolute or relative insulin deficiency and reduced sensitivity of target cells to insulin[1]. Around 1 in 11 adults worldwide now have diabetes Mellitus, 90\% of whom have Type 2 Diabetes Mellitus (T2DM)[2]. According to the 2017 survey by China's National Center for Disease Control and Endocrinology Branch of the Chinese Medical Association, the prevalence rate of T2DM in China was $12.8 \%$. China has become the country with the largest number of diabetes patients worldwide[3]. The complications of diabetes involves systemic tissues and organs, especially the damage to the eyes, kidneys, cardiovascular system and nervous system, which seriously threatens the life quality of patients and brings a heavy burden to the society[4]. 
Gut microbiota is closely related to diabetes. Chinese research team has clarified the differences in gut microbiota between diabetic patients and healthy people at the molecular level by using metagenomic association analysis[5]. A series of studies in Gordon's lab showed that the gut microbiota in patients with Type 2 Diabetes was characterized by a decrease in the abundance of butyrate producing bacteria, such as Bacteroidetes, and an increase in various opportunistic pathogens, such as Firmicutes[6-7]. Larsen found that in the diabetic patients, Firmicutes, including Clostridium, were significantly reduced, while Proteobacteria were more abundant, and this bacterium was positively correlated with blood glucose concentration[8]. From normal glucose tolerance to pre-diabetes and then to the occurrence of T2DM, the relative abundance of Streptococcus continued to decline, while the relative abundance of verrucosa and Proteobacteria showed an opposite trend, indicating that dysregulation of gut microbiota was associated with the progression of T2DM[9]. Some scholars proposed that gut microbiota influenced the occurrence and development of T2DM mainly through mechanisms such as the effect of intestinal energy acquisition[10-12], regulation of fat storage, influence of adipokines[13-16], and regulation of inflammation induced by metabolic endotoxemia[17].

TCM could treat T2DM by regulating disturbance of gut microbiota. The GGQL formula was proved to enrich the abundance of intestinal beneficial bacteria like Clostridium leptum, inhibit the growth of intestinal pathogenic bacteria like Fusobacterium, thus improving permeability of intestinal mucosa and treat T2DM[18]. Another large RCT showed that both metformin and AMC formula significantly alleviated hyperglycemia and hyperlipidemia by shifting gut microbiota structure in diabetic patients. But only AMC formula increased the probiotics group represented by Faecalibacterium spp, therefore showed better effect in improving homeostasis model assessment of insulin resistance (HOMA-IR) and plasma triglyceride[19]. TCM extracts such as berberine also had a good effect in regulating disorder of gut microbiota. Studies showed that berberine could selectively inhibit the growth of Blautia and Allobaculum[20], increased the abundance of butyrate producing bacteria[21], restored the relative abundance of other bacteria species including Bififidobacteria, Bacteroidetes/ Firmicutes, Bacteroides, and decreased the ratio of Ruminococcus gnavus, Ruminococcus schinkii, Lactobacillus acidophilus, Lactobacillus murinus and Lactococcus lactis[22]. A Meta-analysis on TCM improvement of gut microbiota in the treatment of Type 2 Diabetes showed that TCM significantly reduced fasting blood glucose, postprandial blood glucose, and improved insulin resistance. There was also a significant change in the relative abundance of bacteria in the genera Bacteroides[23].

Previous studies found that the RG formula could reduce postprandial blood glucose by inhibiting intestinal a-glucosidase in T2DM patients[24]. Our team also demonstrated that the RG formula could inhibit the expressions of pro-inflammatory factors and pro-apoptotic factors in pancreatic islets, upregulate adiponectin receptors, and reduce the level of serum free fatty acids, thereby alleviating insulin resistance in diabetic rats[25-26]. Whether long-term intervention of RG formula will remodel the structure of gut microbiota, reduce inflammation and improve insulin resistance, is worthy to be explored. Based on the viewpoint of gut microbiota, this study aims to: (1) assess the clinical efficacy of RG formula in adults with T2DM; (2) characterize the influence of RG formula on levels of inflammatory cytokines and structural changes of gut microbiota; (3)analyse the relationship between changes in gut 
microbiota, inflammatory cytokines and clinical assessments, in order to demonstrate the underlying mechanism of TCM treatment in T2DM patients.

\section{Methods}

\subsection{Study design}

This protocol is designed as a randomized and placebo-controlled trial. Participants and investigators are blinded. A total of 60 subjects will be recruited at the Section II of Endocrinology \& Nephropathy department of Dongzhimen Hospital affiliated to Beijing University of Chinese Medicine. They will be randomized in a 1:1 ratio to an experiment group ( $R G$ formula) and a control group (placebo). Patients in both groups are given basic blood glucose control. Only the use of insulin and insulin secretion promoters(such as thiazolidinedione and sulfonylurea) can be allowed as basic control in both groups, which have no significant influence on gut microbiota according to the available research. The trial flow diagram is illustrated in Fig. 1. The SPIRIT Checklist is shown in Supplemental file 1[27].

\subsection{Participants}

\subsubsection{Diagnostic criteria}

The diagnostic criteria for this trial will be set based on the American Diabetes Association(ADA) guidelines in 2018[28]:(1) Patients with typical diabetes symptoms and random blood glucose $\geq$ $11.1 \mathrm{mmol} / \mathrm{L}$ or random venous plasma glucose $\geq 11.1 \mathrm{mmol} / \mathrm{L}$. Typical symptoms of diabetes include polydipsia, polyphagia, polyuria, dysphoria, and weight loss without other triggers; (2) FBG or venous plasma glucose $\geq 7.0 \mathrm{mmol} / \mathrm{L}$, which is defined as no calorie intake for at least 8 hours; (3) PBG after OGTT test $\geq 11.1 \mathrm{mmol} / \mathrm{L}$; (4) $\mathrm{HbA} 1 \mathrm{c} \geq 6.5 \%$.

\subsubsection{Inclusion criteria.}

Patients who meet the following criteria will be considered for enrollment:(1) Age 18-65;(2) Meet the diabetes diagnostic criteria of ADA;(3) FBG is stable at less than $7 \mathrm{mmol} / \mathrm{L}$ and PBG is stable at less than $10 \mathrm{mmol} / \mathrm{L}$.(4) Informed and consented to volunteer. The process of obtaining informed consent shall comply with GCP regulations.

\subsubsection{Exclusion criteria.}

Patients are excluded from participation in the study if any of the exclusion criteria are met: (1) Age > $=65$ years old; (2) Patients with serious heart, liver, kidney and other complications or with severe cataclysmic disease or mental illness; (3) Pregnant or lactating women; (4) Patients who can not be in conjunction with the diet and medication as prescribed; (5) Inflammatory bowel disease; (6) recent use of antibiotics(within three month); (7) Those who refuse to participate in the study or cannot sign the informed consent. 


\subsubsection{Withdrawal criteria.}

Participants who meet any of the following conditions will be removed from the study: (1) Those who cannot adhere to or automatically abandon the prescribed treatment during the study observation; (2) Incomplete data or poor compliance are found during the study observation, which affected the judgment of efficacy; (3) Severe cardiovascular and cerebrovascular complications and infections occurred during the study observation; (4) Serious adverse events occurred during the study observation and it is inappropriate to continue the study;(5) Severe changes of primary disease in the course of observation.

\subsection{Study setting}

The treatment will be conducted at Dongzhimen Hospital affiliated to Beijing University of Chinese Medicine. The study will enroll 60 individuals in total, with 30 individuals in each group. Patients who are included in the study are required to sign an informed consent form and will participate in the study on a voluntary basis. The researchers will obtain written informed consent from each participant before screening. The informed consent form is provided in Supplemental file 2. An overview of specific measurements and time points of data collection are provided in Table 1. 
Table 1

Measurement items and point of data capture

\begin{tabular}{|c|c|c|c|c|c|}
\hline Visit project & $\begin{array}{l}\text { Screening } \\
\text { period/ } \\
\text { baseline }\end{array}$ & Visit 1 & Visit 2 & Visit 3 & $\begin{array}{l}\text { visit } \\
4-6\end{array}$ \\
\hline Visiting time & -7 to 0 days & $\begin{array}{l}\text { Medication } \\
\text { week } 4\end{array}$ & $\begin{array}{l}\text { Medication } \\
\text { week } 8\end{array}$ & $\begin{array}{l}\text { Medication } \\
\text { week } 12\end{array}$ & $\begin{array}{l}\text { follow- } \\
\text { up } \\
\text { week } \\
4,8,12\end{array}$ \\
\hline \multicolumn{6}{|l|}{ Collect basic medical history } \\
\hline Sign informed consent & $\sqrt{ }$ & & & & \\
\hline Fill in general information & $\sqrt{ }$ & & & & \\
\hline $\begin{array}{l}\text { Medical history and treatment } \\
\text { history }\end{array}$ & $\sqrt{ }$ & & & & \\
\hline $\begin{array}{l}\text { Determine inclusion and } \\
\text { exclusion criteria }\end{array}$ & $\sqrt{ }$ & & & & \\
\hline Physical examination & $\sqrt{ }$ & $\sqrt{ }$ & $\sqrt{ }$ & $\sqrt{ }$ & $\sqrt{ }$ \\
\hline $\begin{array}{l}\text { Comorbidity and medication } \\
\text { records }\end{array}$ & $\sqrt{ }$ & $\sqrt{ }$ & $\sqrt{ }$ & $\sqrt{ }$ & $\sqrt{ }$ \\
\hline \multicolumn{6}{|l|}{ Effectiveness observation } \\
\hline Glycosylated Hemoglobin & $\sqrt{ }$ & & & $\sqrt{ }$ & $\sqrt{ }$ \\
\hline Fasting blood glucose & $\sqrt{ }$ & & & $\sqrt{ }$ & $\sqrt{ }$ \\
\hline Postprandial blood glucose & $\sqrt{ }$ & & & $\sqrt{ }$ & $\sqrt{ }$ \\
\hline Fasting insulin & $\sqrt{ }$ & & & $\sqrt{ }$ & \\
\hline Fasting C-peptide & $\sqrt{ }$ & & & $\sqrt{ }$ & \\
\hline inflammatory factors & $\sqrt{ }$ & & & $\sqrt{ }$ & \\
\hline \multicolumn{6}{|l|}{ Other work } \\
\hline Random grouping & $\sqrt{ }$ & & & & \\
\hline Fecal specimen & $\sqrt{ }$ & & & $\sqrt{ }$ & $\sqrt{ }$ \\
\hline liver and kidney function & $\sqrt{ }$ & & & $\sqrt{ }$ & $\sqrt{ }$ \\
\hline Adverse event & $\sqrt{ }$ & $\sqrt{ }$ & $\sqrt{ }$ & $\sqrt{ }$ & $\sqrt{ }$ \\
\hline
\end{tabular}

\subsection{Participant recruitment.}


The trial Subjects will be recruited from the clinic or the ward in the Section II of Endocrinology \& Nephropathy department. Patients recruited by advertisement from other hospitals need to undergo an observation period of 1 week. After the observation, The patients who meet the inclusion criteria will be allowed to enter the clinical trial. The following basic personal information will be collected: sex, age, body mass index (BMI), educational background, occupation, marital status, related past health history, etc. These details about the participants will be maintained by the data monitoring committee (DMC) and will never be revealed to any individual or organization not connected to the study.

\subsection{Randomization and blinding}

This clinical research adopts randomized, double-blind, parallel-group study method. Patients who meet the criterion of Type 2 Diabetes are randomly divided into control group and experimental group. Randomization is realized by using the random number table method, which is generated by Strategic Applications Software by a statistician blinded to the treatment and data collection.

The group name will be written on a card and sealed in an opaque envelope. The sequence numbers will be written on the envelopes, and the envelopes will be numbered sequentially.

The sequence number table should be made in triplicate and kept by the project leader, the sponsor and the data analyst. The seal of envelope should not be broken. When the blind is broken, the three copies of the random assignment table should be unsealed face to face at the same time. All experimental drugs of each subject are put into a drug bag and numbered outside the drug bag $(1,2,3 \ldots)$. According to the sequence number of the random allocation table, different drugs will be loaded into the corresponding drug bag by a third party. In this trial, we use the simulation agent made of dextrin and condiment as the placebo. Both RG formula and placebo are manufactured by

Kang Taisheng Pharmaceutical Co., Ltd. with the same specification, appearance and flavor.The blinding will be monitored and assessed by independent statisticians responsible for data monitoring and by the Ethics Committee of the study center. The blind code will be disclosed after completion of the statistical analysis.

\subsection{Intervention}

Patients in both groups are given diabetes education and lifestyle intervention. After basic blood glucose control, the fasting blood glucose is stable at less than $7 \mathrm{mmol} / \mathrm{L}$ and postprandial blood glucose is stable at less than $10 \mathrm{mmol} / \mathrm{L}$. In this process, metformin, GSDI (a-glucosidase inhibitor), DPP-4(dipeptidyl peptidse 4) inhibitor and GLP-1 (glucagon-likepeptide-1) analogue are avoided. Insulin and insulin secretion promoters are allowed. On the basis of standard treatment, The experimental group is given TCM granule packet. Each packet contains $11 \mathrm{~g}$ RG formula, which include $10 \mathrm{~g}$ coptis root and $1 \mathrm{~g}$ ginseng. Patients will take RG formula at least for 3 months, 2 times a day, $11 \mathrm{~g}$ each time. The control group is given the same amount of Chinese medicine simulation agent which is taken by the same way. The simulation agent contains dextrin and condiment, which looks and tastes exactly like RG formula. The course of intervention is 12 weeks, and medicine is given every 4 weeks. Serum and stool samples 
are collected at baseline and at week 12 for relevant indicators. During the trial, the subjects are forbidden not to use other TCM drugs (including proprietary Chinese medicine, TCM injections, etc.) in combination. For patients who have chronic complicated diseases and need to take drugs for a long time, which do not conflict with the above inclusion and exclusion criteria, their combined diseases and medication conditions shall be recorded in detail in the Case Report Form (CRF). Follow-up observation is continued after 12 weeks of administration to determine the subsequent effects of the drug. This study has been approved by the Ethics Committee of Dongzhimen Hospital and all patients will sign the informed consent after understanding the content, process and significance of the study.

\subsection{Outcome measures}

The physical condition of patients should be recorded at baseline, week 4, week 8 and week 12 .

At the start of the trial, we will collect the patient's general information including name, gender, age, education, marriage condition, $\mathrm{BMI}$ and etc. The complications of diabetes such as diabetic retinopathy, diabetic nephropathy, diabetic peripheral neuropathy and diabetic peripheral vascular disease should be especially recorded. The other diseases, the corresponding treatment, symptoms, tongue and pulse condition of TCM should also be collected. At week 4 and week 8 , two follow-up visits are conducted to collect TCM related indicators such as symptoms, tongue signs, pulse signs and symptoms diagnosis. At week 0 and week 12, fecal samples and blood samples are collected for quantitative determination of gut microbiota, serum biochemical indicators and inflammatory indicators.

\subsubsection{Primary outcomes}

The primary outcomes in this study is the mean change in Glycosylated Hemoglobin(HbA1c) from baseline to 12 weeks intervention and 12 weeks follow-up intervention between the two groups and within groups. HbA1c is a critical indicator for the diagnosis of T2DM, which can reflect average levels of glycemia during the preceding 3 months and is widely used for diabetic complication assessment [29]. It is considered as the gold standard for predicting glycaemia-associated risks for the microvascular and macrovascular complications of diabetes mellitus over 5-10 years[30]. Therefore, The value of $\mathrm{HbA} 1 \mathrm{c}$ in the care of patients with T1DM and T2DM is unassailable[31]. Professional societies, public health organizations, regulatory agencies, patients, and clinicians have focused on $\mathrm{HbA1c}$ levels to gauge the quality of diabetes care and over time, $\mathrm{HbA} 1 \mathrm{c}$ level has supplanted other indicators of the quality of diabetes care, such as blood glucose levels and symptoms of hyperglycemia, despite being a surrogate rather than a direct marker of glycemic control[32]. In addition, careful regulation of blood glucose concentrations in patients with diabetes mellitus normalized HbA1c over 6 weeks[33], so it has also excellent reliability and validity and is sensitive enough for clinical and research practice.

\subsubsection{Secondary outcomes}

The mean changes in FBG, PBG, FIL, C-P, IRI, inflammatory factors and species abundance of gut microbiota before and after 12 weeks treatment are secondary outcomes. FBG, PBG, FIL and C-P will be measured to further assess and compare change in insulin resistance status. PBG is obtained by rapid 
detection of fingertip blood. FBG, FIL and C-P is obtained by venous blood sample which will be collected after an overnight fast by a trained medical officer/investigator and transported to a standard tertiary care laboratory for analysis. Laboratory technicians are blinded to the study participants. The insulin resistance index of homeostasis model is based on the formula developed by Matthews[34]. The inflammatory factors include Interleukin-6 (IL-6), tumor necrosis factor a (TNF-a) and C-reactive protein (CRP), which will be determined by investigator by ELISA. The species abundance of gut microbiota will be based on the second-generation high-throughput sequencing technology, in which the V3-V4 region of $16 \mathrm{~S}$ rRNA gene in the samples is sequenced without isolation and culture of microorganisms. The OTU clustering, species annotation, a-diversity, $\beta$-diversity and LDA Effect Size difference score will be analyzed with bioinformatics analysis method.

\subsubsection{Safety indicator}

Laboratory tests including blood routine and urine routine, liver and kidney function are measured before and after the study. Adverse events, such as hypoglycemic reactions, constipation, and abdominal pain, are recorded in detail and their association with medication is determined. The trial can be terminated if necessary.

\subsubsection{Safety assessment}

During the study period, the Adverse Event Record Form is completed to record the duration, occurrence time, severity, measures and outcome of the adverse event. The severity of the adverse events is divided into mild, moderate and severe. Mild means mild discomfort, which is tolerable and do not require special treatment; moderate is intolerable and require special treatment; severe discomfort require timely termination of the trial and immediate emergency treatment. Researchers will report it to the person in charge of the trial, the sponsoring unit and the medical ethics committee in time, and even report it to the adverse reaction center according to the relevant regulations of the State Medical Products Administration. Ethics committee have the right to make the final decision to terminate the trial.

\subsection{Sample size}

Sixty participants $(n=60)$ will be recruited after run-in period and allocated to RG group $(n=30)$ and placebo group $(n=30)$. The sample size was calculated considering the expected change of glycosylated hemoglobin (HbA1c) (primary outcome) of the participants by software PASS15.0. Previous studies have reported that formula including Coptis Root and Ginseng had statistically and clinically significant reduction of absolute $\mathrm{HbA} 1 \mathrm{c}$ by about $0.72 \%[35,36]$. Sample size was calculated to have $80 \%$ power to detect a moderate $0.65-\mathrm{SD}$ difference in $\mathrm{HbA1c}$, with an alpha/a value of 0.05 . Additional participants will be recruited until the required number is achieved $(n=30)$ per group to account for the estimated drop-out rate of $15 \%$. Therefore, we take the sample size as 30 in both groups.

\subsection{Patient and public involvement}

Patients in this trial will not be involved in the design, recruitment or conduction of the study. After the measure of the samples, the patients will be informed of the results of indicator data in the form of 
report. The doctor in charge of the patient will be also informed of the results to optimize treatment and the rehabilitation scheme.

\subsection{Adherence to study medication}

Strategies to improve adherence to intervention protocols include:

(1)Providing professional lifestyle guidance, 2 weeks of TCM treatment and laboratory index containing liver function, kidney function, $\mathrm{HbA1c}$, fasting blood glucose, postprandial blood glucose and gut microbiota for free. The outcome of HbA1c, fasting blood glucose and postprandial blood glucose are instant available.

(2)Subsidizing the cost of patients' travel to the hospital for visits.

(3)Adding the patient's network contact address, communicating and solving the patients' problems at any time, requiring the patient to monitor blood glucose at home regularly and upload blood glucose data, continuing to conduct diabetes education.

(4)Checking the number of medicine bags left by the patients at each return visit, as well as confirming the situation of medication.

\subsection{Quality control and data collection}

In order to maintain the high quality of the trial and ensure compliance with the protocol, all researchers and drug administrators participating in the study will receive rigorous training in accordance with the standardized operating procedures (SOP) manual. Researchers should accurately and clearly record patients' information in accordance with the requirements of the CRF. The correction of error and its time will be marked. The quality of the study will be managed through the Dongzhimen Hospital Data Monitoring Committee(DMC), where managers regularly check data sheets for omissions and errors to monitor the integrity of test data. The DMC consists of endocrinology clinical experts, methodology experts and biostatisticians, which are independent of the sponsor and have no conflict of interest with research team. Original CRFs will be reserved at the research center in Dongzhimen hospital for 5 years for reference by researchers after completion of the study.

\subsection{Statistical analysis}

SPSS 20.0 software was used for statistical analysis of data. Measurement data were described as mean (M) \pm standard deviation (SD). Normality of continues data was evaluated using Kolmogorov-Smirnov test and Q-Q plot. If the paired samples were in accordance with normal distribution, the t-test of the paired samples was used for pairwise comparison. If not, the nonparametric test was adopted. If the two independent samples were in accordance with normal distribution and homogeneity test of variances, the t-test of independent sample was used. The t'-test was used when variance was not uniform and the nonparametric test was adopted when the samples were in not accordance with normal distribution. Enumeration data like gender and medical history was described statistically by frequency. Chi-square 
test and Wilcoxon rank sum test were used to determine whether there was comparability between the two groups. All statistical tests are bilateral, and a $\mathrm{P}$ value $\leq 0.05$ would be considered statistically significant.

\section{Discussion}

Diabetes greatly impacts the quality of life and health of the patient, the medical costs of its complications have become a heavy burden on individuals and the society. For a long time, the questions of how to effectively treat $\mathrm{DN}$, reduce insulin resistance and delay the progression of complications have always been the focus and difficulty of domestic and foreign research. TCM has the advantages of multiple targets, multiple pathways and few side effects in the treatment of T2DM. With the further research on gut microbiota of T2DM, more and more attention has been paid to the specific effects of TCM on gut microbiota, but among which is lacking the clinical studies. So it remains unclear how TCM treats diabetes by regulating gut microbiota.

Our preliminary research shows that the RG formula can effectively alleviate chronic inflammation, insulin resistance, improve reduce fasting and postprandial blood glucose in rats with T2DM[37]. Therefore, it is necessary to carry out the clinical research to explore the clinical efficacy and safety of drugs. In terms of its toxicology, the specific dose of coptis root and ginseng were both certified as safe. In 2012, researchers concluded that dosage of berberine for oral administration at $20.8 \mathrm{~g} / \mathrm{kg}$ (or a berberine blood concentration of $0.168 \mu \mathrm{g} / \mathrm{mL}$ ) was safe in mice, and the safety dosage for humans would be $2.97 \mathrm{~g} / \mathrm{kg}$ of human body weight, which was much higher than the clinically recommended dosage of $15 \mathrm{mg}$ berberine/ $\mathrm{kg}$ of human body weight[38]. Ginseng had nearly no toxic effects in a regular intake(3-9g)[39]. Only in the case of high dose intake of ginseng (up to $15 \mathrm{~g} /$ day), there can be a small number of adverse reaction[40]. We expect the trial as safe and our findings may provide a new perspective to illustrate the mechanism of TCM treatment and promote the widespread application of it.

\section{Ethics And Dissemination}

The protocol has been approved by the Ethics Committee of Dongzhimen Hospital affiliated to Beijing University of Chinese Medicine (DZMEC-KY-2017-126). The results of the clinical trial will be disseminated through peer-reviewed publications and conferences.

\section{Trial Status}

This study is under participant recruitment. The first patient was included on 12 Feb. 2018, and the recruitment will be finished before 28 August 2021. At present, 44 patients have been recruited.

\section{Declarations}


Acknowledgments We would like to thank the study participants for their time and cooperation, and the staffs in Section II of Endocrinology\&Nephropathy department and the clinical biobank of Dongzhimen Hospital for their collaboration.

Contributors LJ and QF contributed equally in drafting this manuscript. QF prepared the informed consent and finished trial registration. SDW, JXZ and WJH are in charge of trial registration and project administration. YC and JYL are in charge of ethical examination. YHX and RXS are in charge of screening and recruitment. Esther AS and JHW are in charge of data processing and analysis. YFZ and ZL are in charge of reviewing and editing the article. JTL and XZF are in charge of designing the advertisement. YYL, YZ and GXS are in charge of exclusion of participants. All authors discussed, revised and approved of the final manuscript.

Funding This study is supported by grants from The General Project of National Natural Science Foundation of China (No. 81774272), Project for Young Teachers of Beijing University of Chinese Medicine(No.2018-JYBZZ-JS062)

Competing interests None declared.

Ethics approval and consent to participate This study was approved by the Institutional Review Board of the Ethics Committee of Dongzhimen Hospital affiliated to Beijing University of Chinese Medicine. The study coordinator will obtain consent from the eligible patients with Institutional Review Board (IRB)approved consent forms.

Consent for publication Not applicable.

\section{Abbreviations}

T2DM

Type 2 Diabetes Mellitus; RG:Coptis Root; FBG:fasting blood glucose; PBG:postprandial blood glucose; FIL:fasting insulin; C-P:fasting C-peptide; IRI:insulin resistance index; HOMA-IR:homeostasis model assessment of insulin resistance; BMI:body mass index; DMC:data monitoring committee; GSDI:aglucosidase inhibitor; DPP-4:dipeptidyl peptidse 4; GLP-1:glucagon-likepeptide-1; CRF:Case Report Form; IL-6:Interleukin-6; TNF-a:tumor necrosis factor a; CRP:C-reactive protein.

\section{References}

1 Rachdaoui N. Insulin: The Friend and the Foe in the Development of Type 2 Diabetes Mellitus. Int J Mol Sci. 2020;21:1770. 
2 Zheng Y, Ley SH, Hu FB. Global aetiology and epidemiology of Type 2 Diabetes Mellitus and its complications. Nat Rev Endocrinol. 2018;4:88-98.

3 Chinese Diabetes Society. Guidelines for the prevention and control of Type 2 Diabetes in China (2017 Edition).Chinese Journal of Practical Internal Medicine. 2018;38:292-294.

4 Cannon A, Handelsman Y, Heile M, Shannon M. Burden of Illness in Type 2 Diabetes Mellitus. J Manag Care Spec Pharm. 2018;24(9-a Suppl):S5-S13.

5 Qin J,Li Y,Cai Z,et al.A metagenome-wide association study of gut microbiota in Type 2 Diabetes.Nature. 2012;490:55-60.

6 Ley RE,Turnbaugh PJ,Klein S,et al.Microbial ecology: human gut microbes associated with obesity.Nature. 2006;444:1022-1023】

7 Ley RE,B ckhed F,Turnbaugh P,et al.Obesity alters gut microbial ecology.Proc Natl Acad Sci USA. 2005;102:11070-11075区

8 Larsen N,Vogensen FK,van den Berg FW,et al.Gut microbiota in human adults with Type 2 Diabetes differs from non-diabetic adults. Plos One. 2010;5:e9085.

9 Zhang X,Shen D,Fang Z,et al.Human Gut Microbiota Changes Reveal the Progression of Glucose Intolerance.PLOS ONE. 2013;8:e71108.

10 Salgaço MK, Oliveira LGS, Costa GN,et al. Relationship between gut microbiota, probiotics, and Type 2 Diabetes Mellitus. Appl Microbiol Biotechnol. 2019;103(23-24):9229-9238.

11 Le Roith D,Shiloach J,Roth J,et al.Insulin or a closely related molecule is native to Escherichia coli.J Biol Chem. 1981;256:6533-6536》

12 Jumpertz R,Le DS,Turnbaugh PJ,et al.Energy-balance studies reveal associations between gut microbes,caloric load,and nutrient absorption in humans.Am J Clin Nutr. 2011;94:58-65】

13 Xiong Y,Miyamoto N,Shibata K,et al.Short-chain fatty acids stimulate leptin production in adipocytes through the G proteincoupledreceptor GPR41.Proc Natl Acad Sci USA. 2004;

101:1045-1050区

14 Bckhed F,Ding $H$,Wang T,et al.The gut microbiota as an environmental factor that regulates fat storage.Proc Natl Acad Sci USA. 2004;101: 15718-15723区

15 Bckhed F,Manchester JK,Semenkovich CF,et al.Mechanisms Underlying the resistance to diet-induced obesity in germ-free mice.Proc Natl Acad Sci USA. 2007;104:979-984区 
16 Aornsson L,Hunag Y,Parini P,et al.Decreased fat storage by Lactobacillus paraeasei is associated with increased levels of an giopoietin-like4 protein(ANGPTIA).PLoS One. 2010;5:e13087】

17 Cani PD,Amar J,Iglesias MA,et al. Metabolic endotoxemia initiates obesity and insulin resistance.Diabetes. 2007,56(7):1761-1772

18 J. Xu, F. Lian, L. Zhao, et al. Structural modulation of gut microbiota during alleviation of Type 2 Diabetes with a Chinese herbal formula.ISME J. 2015;9:552-562.

19 X. Tong, J. Xu, F. Lian, et al. Structural Alteration of Gut Microbiota during the Amelioration of Human Type 2 Diabetes with Hyperlipidemia by Metformin and a Traditional Chinese Herbal Formula: a Multicenter, Randomized, Open Label Clinical trial.mBio. 2018;22:e02392-17.

20 X. Zhang, Y. Zhao, M. Zhang, et al.Structural changes of gut microbiota during Berberine-mediated prevention of obesity and insulin resistance in high-fat diet-fed rats, PLoS ONE. 2012;7:e42529.

21 Y. Wang, J. Shou, X. Li, et al., Berberine-induced bioactive metabolites of the gut microbiota improve energy metabolism, Metabolism. 2017;12:72-84.

22 Y. Cao, Q. Pan, W. Cai, et al., Modulation of gut microbiota by Berberine improves steatohepatitis in high-fat diet-fed BALB/C mice, Arch. Iran Med. 2016;19:197-203.

23 Y. Zheng, Q. Ding,Y. Wei, et al. Effect of traditional Chinese medicine on gut microbiota in adults with Type 2 Diabetes: A systematic review and meta-analysis. Phytomedicine. 2020;12:153455.

24 T. Shan, J. Sun ,H. Zhang.Effects of ginseng coptis root on a-glucosidase. Jilin Journal of Traditional Chinese Medicine. 2012;32:192-194. in Chinese

25 L.F. Li , S.D. Wang, J.X. Zhao, et al. Effects of RG formula on glucose and lipid metabolism in type 2 diabetic rats with insulin resistance. China Journal of Basic Medicine of Traditional Chinese Medicine. 2006;9:707-708. in Chinese

26 D.Y. Zhou, X. Mu, S.D. Wang, et al. Effects of RG formula on glucose metabolism, islet function and morphology of spontaneous diabetic rats. Chinese Archives of Traditional Chinese Medicine. 2017;35:1702-1704. in Chinese

27 Chan A W, Tetzlaff J M, Gøtzsche P C, et al. SPIRIT 2013 explanation and elaboration: guidance for protocols of clinical trials. BMJ 2013;346:e7586.

28 American Diabetes Association. Glycemic targets: standards of medical care in diabetes-2018. Diabetes Care 2018;41:S55-64.

29 Ribeiro RT, Macedo MP, Raposo JF. HbA1c, Fructosamine, and Glycated Albumin in the Detection of Dysglycaemic Conditions. Curr Diabetes Rev. 2016;12(1):14-9. 
30 Klein, K. R., \& Buse, J. B. The trials and tribulations of determining targets for diabetes mellitus. Nature Reviews.Endocrinology. 2020;16(12):717-730.

31 Syed IA, Khan WA. Glycated haemoglobin--a marker and predictor of cardiovascular disease. J Pak Med Assoc. 2011;61(7):690-695.

32 Rodriguez-Gutierrez, R. \& McCoy, R. G. Measuring what matters in diabetes. JAMA.2019;321(19),18651866.

33 Koenig, R. J. Correlation of glucose regulation and hemoglobin Alc in diabetes mellitus. N. Engl. J. Med. 1976;295(12), 417-420.

34 Matthews D R, Hosker J P, Rudenski A S, et al. Homeostasis model assessment: insulin resistance and $\beta$-cell function from fasting plasma glucose and insulin concentrations in man. Diabetologia. 1985;28(14):412-419.

35 Vuksan, V., Zenith, Z. X., Jovanovski, E., et al. Efficacy and safety of american ginseng extract on glycemic control and cardiovascular risk factors in individuals with type 2 diabetes: A double-blind, randomized, cross-over clinical trial. European Journal of Nutrition. 2019;58(3), 1237-1245.

36 Dong, H., Wang, N., Zhao, L. Berberine in the treatment of type 2 diabetes mellitus: A systemic review and meta-analysis. Evidence - Based Complementary and Alternative Medicine, 2012 doi:http://dx.doi.org/10.1155/2012/591654

$37 \mathrm{~S}$. Wang. Study on the mechanism of the treatment of type 2 diabetes mellitus by the combination of heat-clearing and qi-replenishing traditional Chinese medicine[D].Beijing: Beijing University of Chinese Medicine, 2007:43-49. in Chinese

38 Kheir M. M., Wang Y., Hua L., et al. Acute toxicity of berberine and its correlation with the blood concentration in mice. Food and Chemical Toxicology. 2010;48:1105-1110.

39 Mancuso C, Santangelo R. Panax ginseng and Panax quinquefolius: From pharmacology to toxicology. Food Chem Toxicol. 2017;107(Pt A):362-372.

40 Siegel R.K. Ginseng and high blood pressure. JAMA. 1980;243:32.

\section{Figures}




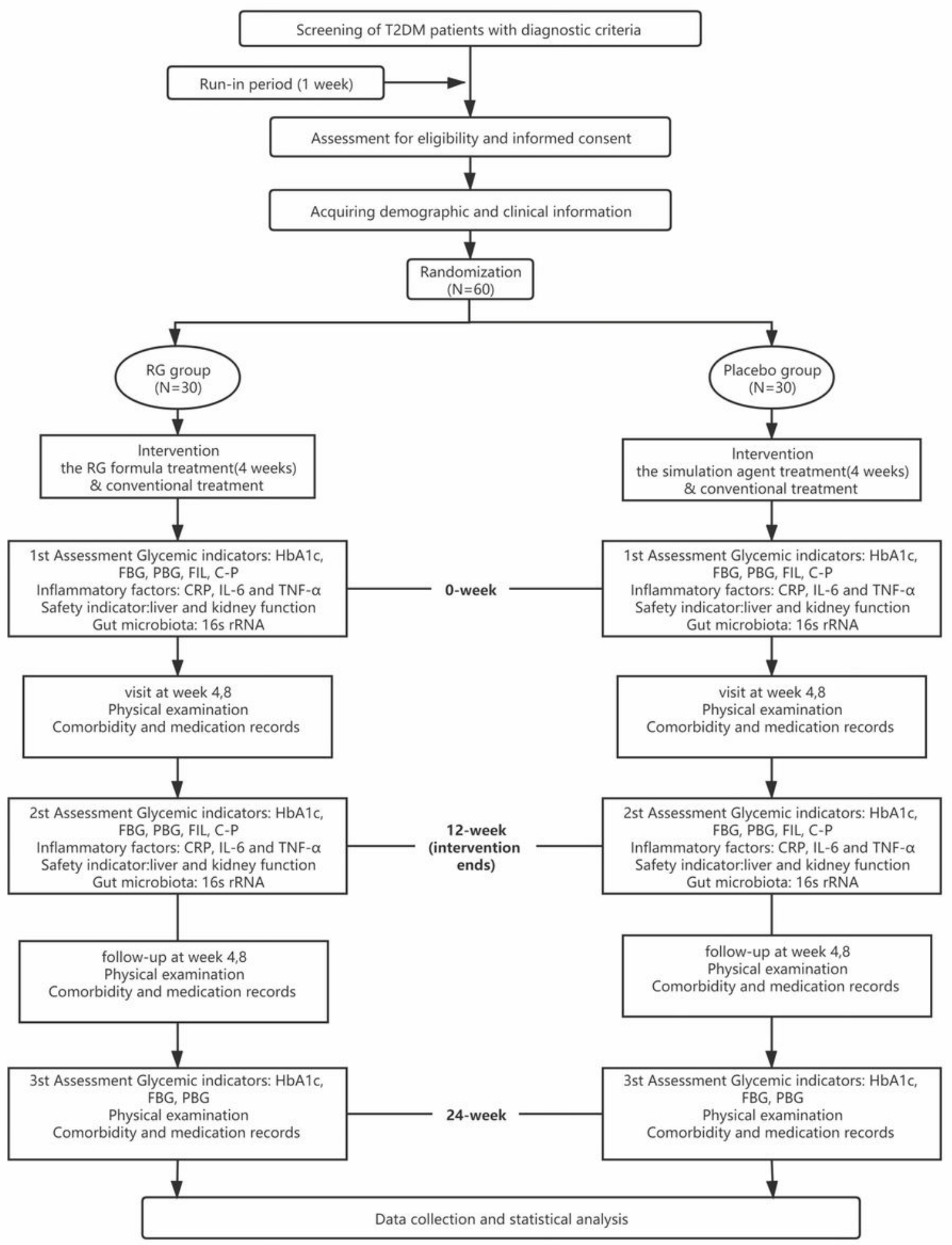

\section{Figure 1}

The trial flow diagram

\section{Supplementary Files}

This is a list of supplementary files associated with this preprint. Click to download. 
- Attachment1spiritstandard.doc

- Attachment2InformedConsent.doc 